\title{
BET inhibitor attenuates heart failure
}

Heart failure (HF) is a leading cause of mortality and hospitalization. Writing in Science Translational Medicine, Duan and colleagues demonstrate that inhibition of bromodomain-containing protein 4 (BRD4), a member of the bromodomain and extraterminal domain (BET) family of epigenetic signalling proteins, reduces pathology and improves outcomes in mouse models of HF, even if administered after the disease is established.

Patients with systolic HF are typically given antagonists of $\beta$-adrenergic receptors and of the

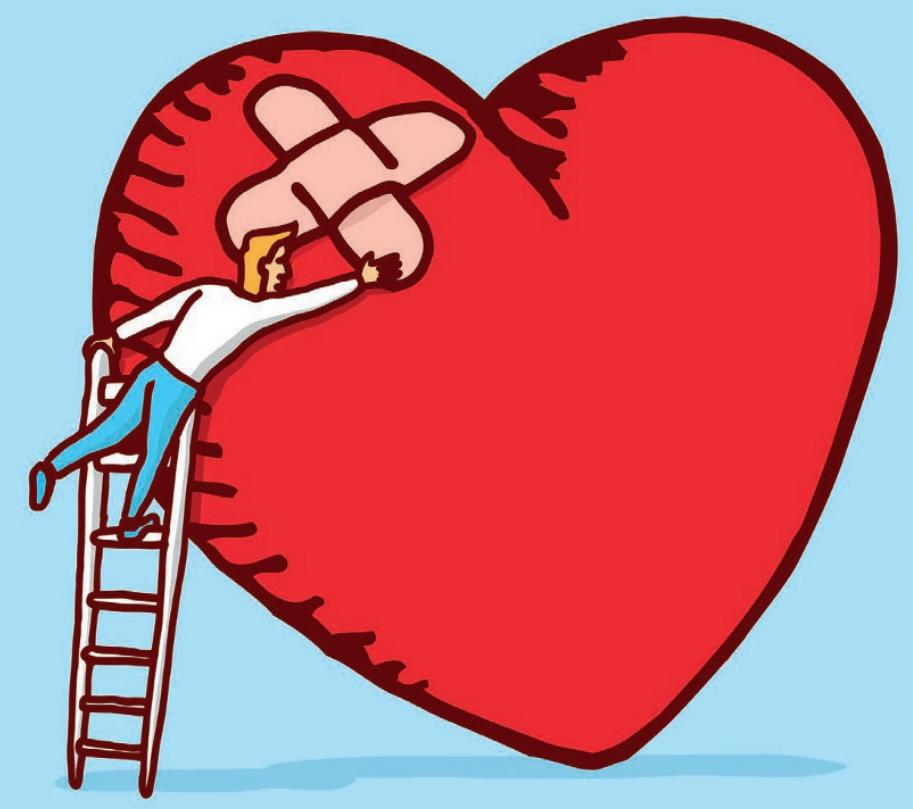

Diego Schtutman/Alamy Stock Photo renin-angiotensin axis, which decrease the levels of stress-induced hormones and also reduce left ventricular (LV) remodelling. However, the 5-year mortality after an initial diagnosis of $\mathrm{HF}$ is $40 \%$, so new treatments are needed. Previous work from this group showed that BRD4 is a critical co-activator of pathologic gene transactivation during cardiomyocyte hypertrophy, and that the BRD4 inhibitor JQ1 could prevent the development of cardiac hypertrophy and LV dysfunction if administered at the onset of pressure overload in mice.

To extend this work, they tested whether JQ1 could treat established disease caused by transverse aortic constriction (TAC), which induces LV pressure overload. After 18 days of TAC, when substantial cardiomegaly and LV dysfunction are apparent, mice received JQ1 (50 mg per kg daily by intraperitoneal injection) or placebo until post-operative week 8 . Compared with placebo, JQ1 reduced signs of HF, including cardiomegaly, pulmonary oedema, LV systolic dysfunction, LV cavity dilation, LV wall thickening, cardiomyocyte hypertrophy and LV fibrosis.

Similarly, treatment of mice with JQ1 starting 5 days after a large transmural myocardial infarction (MI) attenuated key features of MI-induced HF, such as those described for the TAC model. Of note, these effects were also observed in tissues distal to the infarcted myocardium, consistent with an effect of JQ1 on the overall tissue remodelling that occurs following MI.

To gain further insight into the mechanism of action of JQ1, the authors carried out transcriptomic profiling on LV tissue from JQ1-treated mice. JQ1 treatment blocked transactivation of a subset of stress-inducible genes, particularly those involved in the transforming growth factor- $\beta$ (TGF $\beta$ ) and nuclear factor- $\kappa B$ (NF- $\kappa B)$ signalling pathways. NF- $\kappa B$ is activated by many of the stress-induced cytokines involved in innate immunity, and TGF $\beta$ is a known regulator of fibrosis, both of which are central to HF pathogenesis.

These findings in animal models were recapitulated in human induced pluripotent stem cell-derived cardiomyocytes (iPSC-CMs). Treatment of iPSC-CMs with JQ1 attenuated the hypertrophic growth caused by endothelin 1 (ET1) and dampened the induction of a subset of ET1induced genes. These genes include those involved in inflammatory responses, cellular growth and extracellular matrix production. Consistent with the in vivo results, genes induced by TGF $\beta$ or NF- $\kappa \mathrm{B}$ were preferentially downregulated by JQ1 treatment in iPSC-CMs.

Although JQ1 is a well-established investigational inhibitor of BRD4, its pharmacological properties are not optimal for clinical use. Many other BET inhibitors, including derivatives of JQ1, are in clinical trials for cancer, which could lay the groundwork for the use of BET inhibitors in HF.

Megan Cully

ORIGINAL ARTICLE Duan, Q. et al. BET bromodomain inhibition suppresses innate inflammatory and profibrotic transcriptional networks in heart failure. Sci. Transl Med. 9 , eaah5084 (2017)

FURTHER READING Filippakopoulos, P. \& Knapp, S. Targeting bromodomains: epigenetic readers of lysine acetylation. Nat. Rev. Drug Discov. 13, 337-356 (2014) 